\title{
EVALUATION OF A PC-BASED DATA ACQUISITION SYSTEM FOR LONG-TERM RECORDING OF ACTION POTENTIALS WITH MINIMAL DEDICATED CIRCUITS
}

\author{
Jen-Yu Li ${ }^{1}$, Jin-KaE JAnG ${ }^{1}$, Te-Son KuO ${ }^{1,2}$, Fu-Shan JaW ${ }^{2}$ \\ ${ }^{1}$ Institute of Electrical Engineering, \\ ${ }^{2}$ Institute of Biomedical Engineering, National Taiwan University, Taipei, Taiwan
}

\begin{abstract}
Owing to the improvement of computing power, computer-aided multi-unit acquisition and separation rapidly proliferated during the last two decades. To utilize this technology is, however, not easy since task-specific designs are usually mandatory. To overcome this obstacle, a commercial data acquisition system was used to evaluate whether it is possible to accomplish the task of multi-unit acquisition without the aid of these devices. As the results shown that the technique of spike-trigger acquisition can provide the capability to reduce the data amount to $2 \%$ while compared with that sampled by continuous acquisition. However, the interval between two consecutive spikes cannot be shorter than $5 \mathrm{~ms}$. This suggests that special designed devices are still necessary.
\end{abstract}

Biomed Eng Appl Basis Comm, 2005(October); 17: 263-266.

Keywords:Action Potential; Data Acquisition; First-In-First-Out Buffer; Multi-unit recording; Personal Computer

\section{INTRODUCTION}

Multi-unit recording is an important method for studying the function of neurons. Conventional techniques often require time-consuming manual intervention [1]. When computers become available to neurophysiologists, various algorithms and systems are developed for online detection and spike separation [23]. Most of them use analog or digital pre-processors to carry out units classification [4-6]. However, these systems are often complicated and require specialized hardware and software, which means one has to make a great effort to construct such systems. On the other

Received: May 3, 2005; Accepted: Aug 20, 2005

Correspondence: Fu-Shan Jaw, Professor

Institute of Biomedical Engineering, National Taiwan University, 5F, IBME, No. 1, Sec. 4, Roosevelt Rd., Taipei, Taiwan 106

E-mail: jaw@ha.mc.ntu.edu.tw hand, using commercial systems specific for this task is an alternative solution. There are currently commercial systems designed to record, store and analyze neural data, such as the products from Plexon Inc. (Texas, USA) or Axon Instruments (California, USA). However, these systems are often too expensive (at least 60,000 USD) to be affordable by laboratories with limited financial budgets.

Recently, many commercial data acquisition cards are very powerful and can be engaged to reduce loading of computers. Therefore, in this study, we tried to examine whether it is possible to implement a system only utilizing hardware and software commonly available. The limitation of this proposed system was evaluated quantitatively and its possible causes were discussed. This information may be valuable for other researchers who may need to build a similar system. 


\section{METHOD}

\subsection{Requirements on the A/D card}

An example of multi-unit signal is shown in Fig. 1. The characteristics of action potentials (APs) put strict requirements on the specification of data acquisition cards [7]. Firstly, the minimal sampling rate of the analog-to-digital (A/D) converter should be 50 $\mathrm{kHz}$ or higher to avoid aliasing errors [8]. Furthermore, the resolution should be no less than 12 bits because the peak amplitude is a critical parameter for unit classification. Moreover, under persistent high-speed acquisition, the amount of data could be massive and difficult to handle. Hence, utilizing the technique of spike-trigger acquisition may resolve this problem. This method can preserve the waveform detail of APs and their time of occurrence for later analysis (see in Fig. 1). Spikes are detected and sampled when a predetermined threshold level is exceeded. The waveforms are then saved in the computer memory together with their time of occurrence indicated by a counter/timer circuit on the A/D card.

As shown in Fig.1, the complete waveform of an AP usually occupies a period from $0.5 \mathrm{~ms}$ before to 2 $\mathrm{ms}$ after its peak amplitude. Thus, a good solution is to incorporate an analog delay line before the $\mathrm{A} / \mathrm{D}$ card so that the pretrigger portion of the spike would not be lost [9]. Instead of using a stand-alone analog delay line, a built-in First-In-First-Out (FIFO) memory can be used to temporarily hold the sampled pretrigger data and provides an alternative solution for this problem.

\subsection{Implementation of the system}

To fulfill all the requirements described above, a high-speed high-resolution A/D card with onboard trigger, timer, and FIFO capabilities is required. A data acquisition card, PCI-6071E (form National Instruments) that met all the requirements was chosen. This card features 64 channels each with 12-bit resolution, 2 analog-output channels, 8 digital I/O lines, two 24-bit counter/timers, 512 words FIFO buffer, and the onboard analog triggering capability. The overall maximal sampling rate can be as high as 1.25 MHz. A shielded rack-mount connecting box, BNC-2090, a shielded cable, SH1006868 (both from National Instruments), and an IBM PC compatible personal computer (Pentium III, $1 \mathrm{GHz}$ ) were used to construct the system.

Application programs for long-term acquisition, storage and waveform display were developed within the LabVIEW (National Instruments) environment. The onboard triggering circuit enables the program to perform spike-trigger acquisition without additional hardware circuits. The $\mathrm{A} / \mathrm{D}$ card was programmed to acquire 25 pretrigger data at $50 \mathrm{kHz}$ in free running mode of operation. The continuously streaming data were temporarily accumulated in the onboard FIFO memory. IF no trigger event occurred, pretrigger samples continuously flew in and out of the FIFO memory. While a spike exceeded the threshold level, the 25 pretrigger samples and the following 100 samples were then taken to compose the complete waveform of the spike. A total of 125 samples was saved along with the time of occurrence onto the hard disk.

\section{RESULT AND DISCUSSION}

\subsection{APs sampling}

Thalamic APs stored on a magnetic tape were used to verify the functions of detection, timing, and data storage of the designed system. In other words, these APs were sampled and saved by utilizing the spike-trigger method. For comparison, the same period of the tape was replayed and continuously sampled by the same system but not in the spike-trigger mode. Fig. 2 shows the results. The upper traces are the continuously sampled waveforms, while the lower traces are the reconstructed APs from the spike-trigger acquisition. For detailed waveform inspection, one of the 4 APs in Fig. 2(b) is displayed in Fig. 2(c) with high resolution. As shown in Fig. 2(c), there is no detectable difference between the 2 waveforms and their valleys are also coincident in time. However, as

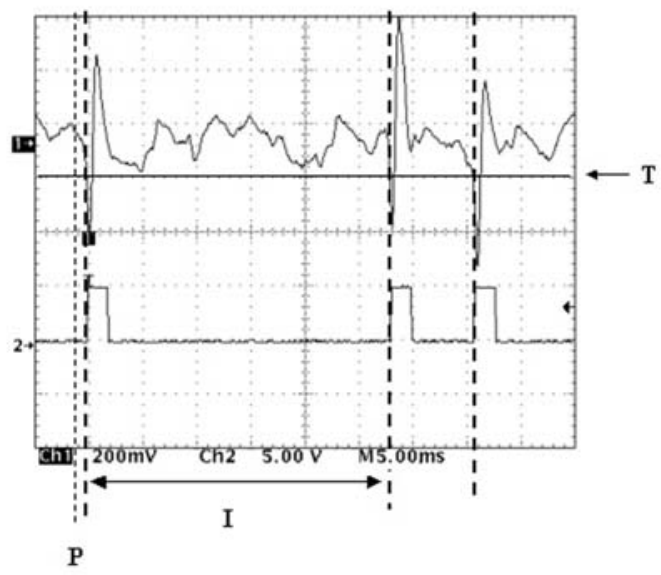

Fig. 1. Illustration of spike-trigger acquisition. The multi-unit recording is shown in the upper trace and the lower trace is the output TTL pulses from a window discriminator. The duration of the TTL pulse is set to $2 \mathrm{~ms}$. The spikes leads the corresponding pulses about $0.5 \mathrm{~ms}$. T: threshold level; I: interspike interval; $P$ : pretrigger portion of the spike. 
we go through the entire record with careful examination, a strange phenomenon was found. As shown in Fig. 2(d), while the interspike interval is shorter than some critical value the following APs may be 'lost' by the spike-trigger method. This is a serious defect that deserves further investigation. Without solving this problem, the proposed system will become useless.

\subsection{Limitation of this system}

To study this problem quantitatively, a precision function generator (modal 95, Wavetek) was used to provide various pulse trains for the simulation of APs. Pulse trains with an interval ranging from 1 to $10 \mathrm{~ms}$ were used to test the spike-trigger acquisition system. The system, including the A/D card and the LabVIEW program, digitized a pulse train and then the interpulse intervals were calculated. Several cycles of pulse trains were used to obtain the mean and standard deviation of the measured intervals, as shown in Fig 3. The horizontal axis is the actual interval of the pulse train, whereas the vertical is the calculated result. When the interpulse interval is larger than or equal to $5 \mathrm{~ms}$, the calculated interval matched the setting of the function generator quite well. On the other hand, if it is shorter than $5 \mathrm{~ms}$, the reconstructed interval showed errors. This figure shows the limitation of our system for spike-trigger acquisition.

For further evaluation of the source of this error, we first replaced the Pentium III PC with a Pentium IV $(2.4 \mathrm{GHz})$ one, and then repeated the evaluation again.
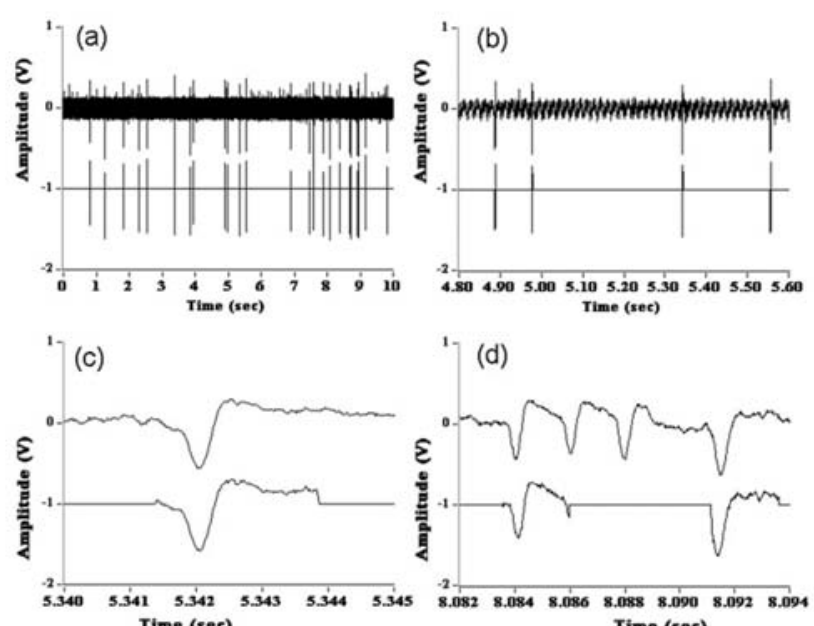

Fig. 2. Comparison of continuously sampled APs (upper traces) and the spike-trigger sampled APs (lower traces) with increasing time resolution from (a) to (c). (d) Two APs are missed by the spiketrigger method.

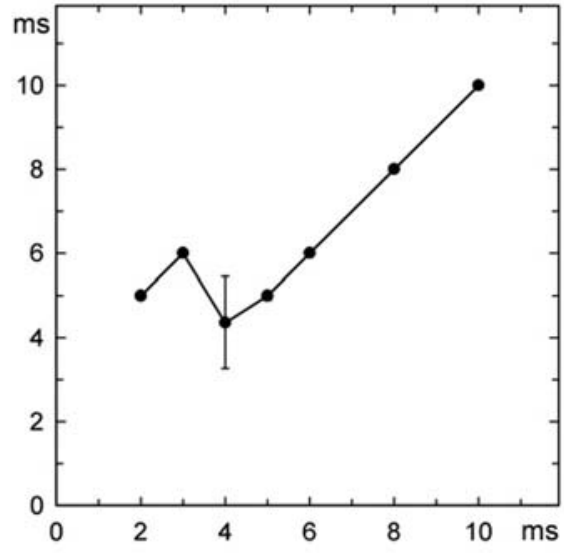

Fig. 3. Effects of the interpulse interval on the spike-trigger method. The shortest interpulse interval by using this system is about $5 \mathrm{~ms}$. Unpredictable results could happen if this limit is violated.

Secondly, another A/D card, PCI-MIO-16E-4 (from National Instruments) was used for evaluation. Unfortunately, all these combination cannot overcome this problem; the minimal $5 \mathrm{~ms}$ interpulse interval cannot be shortened at all. A possible cause of this problem could be due to the LabVIEW program or the Windows environment. The technical detail, however, is outside the scope of our ability. Hence, we had written a letter to the National Instruments for technical support. The returned answer is that this limitation is inherent to the $\mathrm{A} / \mathrm{D}$ card or the LabVIEW itself.

For computerized spikes classification, the spiketrigger acquisition sheds light on the difficulty of data storage and signal processing. To achieve the function of spike-trigger, the analog signal input to the A/D card should be appropriately delayed to obtain the pretrigger portion of a spike. In principle, the multiunit recording can be delayed either analogously or digitally. The purpose of this study is to determine whether we can make use of the onboard resource such as the FIFO memory of the A/D card to replace an analog delay line. The answer, unfortunately, is unsatisfactory. A possible reason could be the overhead for the LabVIEW environment. Anyway, it does not seem workable at present to accomplish the task of multi-unit acquisition period without the aid of specialized instruments. 


\section{ACKNOWLEDGMENT}

This work was supported by grants $92-\mathrm{EC}-17-\mathrm{A}-$ 05S1-0017 from the Ministry of Economic Affairs, Taiwan.

\section{REFERENCE}

1. Schmidt EM: Instruments for sorting neuroelectric data: a review. J Neurosci Methods 1984; 12: 1-24.

2. Lewicki MS: A review of methods for spike sorting: the detection and classification of neural action potentials. Network-Comp Neural 1998; 9: R53-R78.

3. Turner DA and Schlieckert M: Data Acquisition and Analysis System for Intracellular Neuronal Signals. J Neurosci Methods 1990; 35: 241-251.

4. Worgotter F, Daunicht WJ and Eckmiller R: An Online Spike Form Discriminator for Extracellular Recordings Based on An Analog Correlation Technique. J Neurosci Methods 1986; 17: 141-151.

5. Kreiter AK, Aertsen AMHJ and Gerstein GL: A Low-Cost Single-Board Solution for Real-Time, Unsupervised Waveform Classification of Multineuron Recordings. J Neurosci Methods 1989; 30: 59-69.

6. Gadicke R and Albus K: Real-Time Separation of Multineuron Recordings with A Dsp32C Signal Processor. J Neurosci Methods 1995; 57: 187-193.

7. Jaw FS and Wang CY: Notes on the applications of AD card for biomedical signals. Biomed Eng Appl Basis Comm 1993; 5: 773-777.

8. Jaw FS: Optimal sampling of electrophysiological signals. Neurosci Res Comm 2001; 28: 75-84

9. Aldridge JW, Walden JL and Gilman S: Enhancing High-Speed Digitization of Single-Unit NeuronalActivity on A Microcomputer Using A Hybrid Software Hardware Technique. J Neurosci Methods 1989; 28: 205-208. 